\title{
The Signed $(|G|-1)$ subdomination number of Product Graphs
}

\author{
Wei $\mathrm{Shi}^{1,{ }^{*}}$, and Suichao $\mathrm{Wu}^{2}$ \\ ${ }^{1}$ Sanda University, Shanghai, China \\ ${ }^{2}$ School of Mathematics, Physics and Statistics, Shanghai University of Engineering Science, \\ Shanghai, China
}

\begin{abstract}
In this paper, the singed $(|G|-1)$ - subdomination number of product graphs $G$, such as $P_{2} \times P_{n}, P_{2} \times C_{n}, P_{3} \times P_{n}$, are determined by classified discussion and exhaustived method.
\end{abstract}

\section{Introduction}

For terminology and notation not defined here we refer to [1].

Let graph $G=(V, E)$ with vertex set $V$ and edge set $E$, the order of $G$ is denoted by $|G|$. For a vertex $u \in V(G)$, the open neighborhood of $u$ is $N_{G}(u)=\{v \in(G) \mid u v \in E(G)\}$, and the closed neighborhood of $u$ is $N_{G}[u]=N_{G}(u) \cup\{u\} . N_{G}(u)$ and $N_{G}[u]$ are abbreviated by $N(u)$ and $N[u]$ when no confusion is possible.

Given graphs $G_{1}=\left(V_{1}, E_{1}\right)$ and $G_{2}=\left(V_{2}, E_{2}\right)$. The Caresian product $G_{1} \times G_{2}$ of two graphs $G_{1}$ and $G_{2}$, is a graph with vertex set $V\left(G_{1} \times G_{2}\right)=V_{1} \times V_{2}$, edge set $E\left(G_{1} \times G_{2}\right)=\left\{\left(u_{1}, v_{1}\right)\left(u_{2}, v_{2}\right) \mid u_{1}=u_{2}\right.$ and $v_{1} v_{2} \in E\left(G_{2}\right)$, or $v_{1}=v_{2}$ and $\left.u_{1} u_{2} \in E\left(G_{1}\right)\right\} \quad$. Let $f: V \rightarrow\{-1,1\}$, we define $f[u]=\sum_{v \in N[u]} f(v)$. If for every $u \in V(G)$, where $f[u] \geq 1$, then the function $f$ is called a signed dominating function of $G . f(V)$ is defined as $f(V)=\sum_{u \in V} f(u)$. The signed domination number of graph $G$ is defined as $\gamma_{s}(G)=\min \{f(V) \mid f$ is a signed dominating function of $G\}$. For an integer $1 \leq k \leq|G|$, a signed $k$-subdominating function of $G$ is a function $f: V \rightarrow\{-1,1\}$ such that $f[u] \geq 1$

For at least $k$ vertices $u$ of $V(G)$. The signed $k$-subdomination number of graph $G$ is defined as $\gamma_{s}^{k}(G)=\min \{f(V) \mid f$ is a signed k-subdominating function of $G$.

Since the signed domination number of graphs has a wide application background, for example, the establishment of transportation posts and material supply points, etc., and the

\footnotetext{
* Corresponding author: $\underline{\text { w.x.8.8,163.com }}$
} 
calculation of the $k$ - subdomination number is NP complete problem, so the study of domination number of graphs has positive significance.

We number the vertices $u_{i}(i=1,2, \cdots, m)$ of a path $P_{m}$ from left to right, number the vertices $v_{j}(j=1,2, \cdots, n)$ of a cycle $C_{n}$ counter clockwise from vertex $v_{1}$. In this paper, we are interested in signed $(2 n-1)$ - subdomination number of product graph $P_{2} \times P_{n}$ and $P_{2} \times C_{n}$, signed $(3 n-1)$ - subdomination number of product graph $P_{3} \times P_{n}$.

\section{Signed $(2 n-1)$ - subdomination number of graph $P_{2} \times P_{n}$}
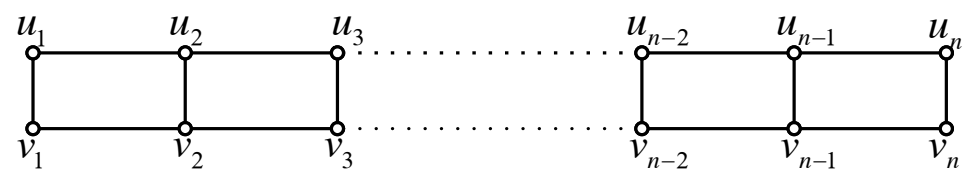

Fig. 1. Graph $P_{2} \times P_{n}$.

Let $f$ be a signed $(2 n-1)$ - subdominating function of $G$. We have the following results.

Lemma 2.1 If vertex $u \in P_{2} \times P_{n}$, where $f[u] \leq 0$, then:

(1) $u$ can't be $u_{1}, v_{1}, u_{n}$ or $v_{n}$.

(2) If $u=u_{i}(2 \leq i \leq n-1)$, then $f\left(u_{i}\right)=1, f\left(u_{i-1}\right)=f\left(u_{i+1}\right)=-1$, and $f\left(v_{i}\right)=1$. Similarly, $u=v_{i}(2 \leq i \leq n-1)$.

Proof. (1) Suppose $u=u_{1}$, where $f\left[u_{1}\right] \leq 0$.

Case 1. If $f\left(u_{1}\right)=-1$, then $f\left(v_{1}\right)=f\left(u_{2}\right)=1$. Otherwise, if $f\left(v_{1}\right)=-1$, then $f\left[v_{1}\right]=f\left(u_{1}\right)+f\left(v_{1}\right)+f\left(v_{2}\right) \leq-1$, contradicting the fact that $f$ is a signed $(2 n-1)$ - subdominating function of $P_{2} \times P_{n}$. So $f\left(v_{1}\right)=1$. Similarly, $f\left(u_{2}\right)=1$. Then, we have $f\left[u_{1}\right]=f\left(u_{1}\right)+f\left(v_{1}\right)+f\left(u_{2}\right)=1$, it is a contradiction.

Case 2. If $f\left(u_{1}\right)=1$. To make $f\left[u_{1}\right]=f\left(u_{1}\right)+f\left(v_{1}\right)+f\left(u_{2}\right) \leq 0$, then $f\left(v_{1}\right)=f\left(u_{2}\right)=-1$, so $f\left[v_{2}\right]=f\left(v_{1}\right)+f\left(v_{2}\right)+f\left(v_{3}\right)+f\left(u_{2}\right) \leq 0$, contradicting the fact that $f$ is a signed $(2 n-1)$ - subdominating function of $P_{2} \times P_{n}$.

Conclusion of Case 1 and 2, $u$ can't be $u_{1}, v_{1}, u_{n}$ or $v_{n}$.

(2) Let $u=u_{i}$, where $f\left[u_{i}\right] \leq 0 \quad(2 \leq i \leq n-1)$. Similarly as Case 1 in (1), we have $f\left(u_{i}\right)=1$. Since $f\left[u_{i}\right]=f\left(u_{i-1}\right)+f\left(u_{i}\right)+f\left(u_{i+1}\right)+f\left(v_{i}\right) \leq 0$, so there are at least two vertices of $\left\{u_{i-1}, u_{i+1}, v_{i}\right\}$, which are adjacent to $u_{i}$, whose values are assigned -1 .

Case 1. If $f\left(u_{i-1}\right)=f\left(u_{i+1}\right)=f\left(v_{i}\right)=-1$, we have $f\left[v_{i-1}\right]=f\left(v_{i-2}\right)+f\left(v_{i-1}\right)$ $+f\left(v_{i}\right)+f\left(u_{i-1}\right) \leq 0 \quad$ and $\quad f\left[v_{i+1}\right]=f\left(v_{i}\right)+f\left(v_{i+1}\right)+f\left(v_{i+2}\right)+f\left(u_{i+1}\right) \leq 0$, contradicting the fact that $f$ is a signed $(2 n-1)$ - subdominating function of $P_{2} \times P_{n}$. 
Case 2. If $f\left(u_{i-1}\right)=1, f\left(u_{i+1}\right)=f\left(v_{i}\right)=-1$, we have $f\left[v_{i+1}\right]=f\left(v_{i}\right)+f\left(v_{i+1}\right)$ $+f\left(v_{i+2}\right)+f\left(u_{i+1}\right) \leq 0$, contradicting the fact that $f$ is a signed $(2 n-1)$ - subdominating function of $P_{2} \times P_{n}$. Similarly, if $f\left(u_{i-1}\right)=f\left(v_{i}\right)=-1, f\left(u_{i+1}\right)=1$, a contradiction too.

In conclusion, the result is true.

Next, we give the result about the singed $(2 n-1)$-subdomintion number of graph $P_{2} \times P_{n}$.

Theorem 2.2 For any integer $n(n \geq 3), \gamma_{s}^{2 n-1}\left(P_{2} \times P_{n}\right)=2 n-2\left[\frac{2 n+3}{4}\right]$.

Proof. Note that, if $n=2$, there are only four vertices in graph $P_{2} \times P_{2}$. By Lemma 2.1, all of them cannot be $u$, where $f[u] \leq 0$. Therefore, it is only when $n \geq 3$ that we can define the signed $(2 n-1)$-dominating function of graph $P_{2} \times P_{n}$.

Let $f$ be the minimal signed $(2 n-1)$ - subdominating function, that is, $f\left(P_{2} \times P_{n}\right)=\gamma_{s}^{2 n-1}\left(P_{2} \times P_{n}\right)$, denote $P=\left\{v \in P_{2} \times P_{n} \mid f(v)=1\right\} \quad$ and $\quad M=\left\{v \in P_{2} \times P_{n} \mid\right.$ $f(v)=-1\}$, the order of them is denoted by $p=|P|, m=|M|$, where $p+m=2 n$. The number of edges between two sets is denoted by $e(P, M)$.

Notice that, $f$ is a signed $(2 n-1)$ - subdominating function of $P_{2} \times P_{n}$. According to Lemma 2.1 , only one vertex of $P$ is adjacent to two vertices of $M$, the last $p-1$ vertices are adjacent to at most one vertex of $M$. So $e(P, M) \leq p+1$. For a vertex of $M$, if it is located at a corner, then it must be adjacent to two vertices in $P$, otherwise it is adjacent to three vertices in $P$. It implies that $e(P, M) \geq 3 m-2$. Since $3 m-2 \leq e(P, M) \leq p+1$, so $2 n=p+m \geq 4 m-3$, then we have $m \leq \frac{2 n+3}{4}$, namely $m \leq\left[\frac{2 n+3}{4}\right]$. Then, we have $\gamma_{s}^{2 n-1}\left(P_{2} \times P_{n}\right)=2 n-2 m \geq 2 n-2\left[\frac{2 n+3}{4}\right]$.

Otherwise, the signed $(2 n-1)$-subdominating function $g$ of $P_{2} \times P_{n}$ is defined as follows:

(i) If $n=4 k-1$, let $A=\left\{u_{1}, u_{3}, v_{5}, \cdots, u_{4 i-1}, v_{4 i+1}, \cdots, u_{n}\right\}$, it implies that $|A|=\frac{n-1}{2}+1=2 k=\left[\frac{2 n+3}{4}\right]$;

(ii) If $n=4 k$, let $A=\left\{u_{1}, u_{3}, v_{5}, \cdots, u_{4 i-1}, v_{4 i+1}, \cdots, u_{n-1}\right\}$, it implies that $|A|=\frac{n}{2}=2 k=\left[\frac{2 n+3}{4}\right]$;

(iii) If $n=4 k+1$, let $A=\left\{u_{1}, u_{3}, v_{5}, \cdots, u_{4 i-1}, v_{4 i+1}, \cdots, v_{n}\right\}$, it implies that $|A|=\frac{n-1}{2}+1=2 k+1=\left[\frac{2 n+3}{4}\right]$;

(iv) If $n=4 k+2$, let $A=\left\{u_{1}, u_{3}, v_{5}, \cdots, u_{4 i-1}, v_{4 i+1}, \cdots, v_{n-1}\right\}$, it implies that $|A|=\frac{n}{2}=2 k+1=\left[\frac{2 n+3}{4}\right]$.

Let $g(v)=\left\{\begin{array}{ll}-1, & \text { if } \mathrm{v} \in \mathrm{A} ; \\ 1, & \text { otherwise, }\end{array}\right.$ then $\gamma_{s}^{2 n-1}\left(P_{2} \times P_{n}\right) \leq 2 n-2\left[\frac{2 n+3}{4}\right]$. 
Consequently, the equality holds.

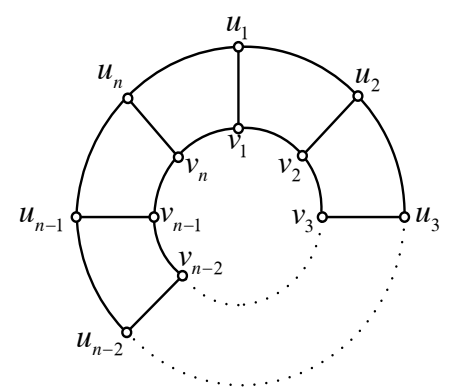

Fig. 2. Graph $P_{2} \times C_{n}$.

\section{Signed $(2 n-1)$ - subdomination number of graph $P_{2} \times C_{n}$}

Theorem 3.1 For any integer $n \quad(n \geq 5)$,

$$
\gamma_{s}^{2 n-1}\left(P_{2} \times C_{n}\right)= \begin{cases}n+2, & \text { if } n \equiv 0(\bmod 4) ; \\ 2 n-2\left[\frac{n}{2}\right], & \text { otherwise. }\end{cases}
$$

Proof. In graph $P_{2} \times C_{n}$, every vertex is adjacent to three vertices. Let $f$ be the minimal signed $(2 n-1)$ - subdominating function, that is, $f\left(P_{2} \times C_{n}\right)=\gamma_{s}^{2 n-1}\left(P_{2} \times C_{n}\right)$. Suppose that $u_{i} \in P_{2} \times C_{n}$, where $f\left[u_{i}\right] \leq 0$. It follows from a same argument as that for Lemma 2.1 that $f\left(u_{i}\right)=1, f\left(u_{i-1}\right)=f\left(u_{i+1}\right)=-1, f\left(v_{i-1}\right)=f\left(v_{i}\right)=f\left(v_{i+1}\right)=1$, where, the index $i$ is understood to be taken modulo $n$. Moreover, to ensure that there exsists a unique $u_{i} \in P_{n} \times C_{n}$ such that $f\left[u_{i}\right] \leq 0$, there must be at least two vertices $u^{\prime}, u^{\prime \prime}$, lying between $u_{i-1}$ and $u_{i+1}$ but not along the direction of $u_{i}$, and two corresponding vertices $v^{\prime}, v^{\prime \prime}$ such that $f\left(u^{\prime}\right)=f\left(u^{\prime \prime}\right)=f\left(v^{\prime}\right)=f\left(v^{\prime \prime}\right)=1$. Therefor, we define the signed $(2 n-1)$-subdominating function of graph $P_{2} \times C_{n}$ with $n \geq 5$.

Denote $P=\left\{v \in P_{2} \times C_{n} \mid f(v)=1\right\}$ and $M=\left\{v \in P_{2} \times C_{n} \mid f(v)=-1\right\}$, the order of them is denoted by $p=|P|, m=|M|$, where $p+m=2 n$. The number of edges between two sets is denoted by $e(P, M)$. In $M$, every vertex is adjacent to three vertices of $P$, so $e(P, M) \geq 3 m$. In $P$, only one vertex is adjacent to two vertices of $M$, the last $p-1$ vertices are adjacent to one vertex of $M$, so $e(P, M) \leq p+1$, then $3 m \leq e(P, M) \leq p+1$, so $2 n=m+p \geq 4 m-1, m \leq \frac{n}{2}+\frac{1}{4}$, therefore $m \leq\left[\frac{n}{2}\right]$.

Next, we will prove that $m \leq\left[\frac{n}{2}\right]-1$ with $n=4 k \quad(k \geq 2)$. 
Proof by contradiction. Suppose $m>\left[\frac{n}{2}\right]-1$, but the integer $m$, where $m \leq\left[\frac{n}{2}\right]$. So $m=\left[\frac{n}{2}\right]=2 k(k \geq 2)$. Let $f\left[u_{i}\right] \leq 0$, that $f\left(u_{i}\right)=1, f\left(u_{i-1}\right)=f\left(u_{i+1}\right)=-1$,

$f\left(u_{j}\right)=f\left(v_{k}\right)=1 \quad(j=i \pm 2, i \pm 3 ; k=i, i \pm 1, i \pm 2)$, where, the index is understood to be taken modulo $n$.

Let set $B=\left\{u_{i+4}, v_{i+3}, v_{i+4}, v_{i+5}\right\}$, that there is only one vetex $v$, where $f(v)=-1$. If there is $f(v)=1$ for any vertex $v \in B$, then, for $v_{i+3}$ and the adjacent three vertices $u_{i+3}, v_{i+2}$ and $v_{i+4}$, that $f\left[v_{i+3}\right]=f\left[u_{i+3}\right]=f\left[v_{i+2}\right]=f\left[v_{i+4}\right]=4$, contrading the fact that $f$ is the minimal signed $(2 n-1)$ - subdominating function. Furthermore, if there is $f\left(v^{\prime}\right)=f\left(v^{\prime \prime}\right)=-1$ for $v^{\prime}, v^{\prime \prime} \in B$, it implies that $f\left[u_{i+4}\right] \leq 0$, contrading the fact that $f$ is the minimal signed $(2 n-1)$ - subdominating function. Therefor, there is only one vertex $v \in B$, where $f(v)=-1$.

Let set $A_{i}=\left\{u_{i}, v_{i}\right\}(1 \leq i \leq n)$. In [3], there is a result:

$$
\left|\left(A_{i} \cup A_{i+1} \cup \cdots \cup A_{i+t}\right) \cap M\right| \leq\left[\frac{t+1}{2}\right]
$$

Discuss the following:

Case 1. If $f\left(v_{i+5}\right)=-1$, then $f\left(u_{i+5}\right)=f\left(u_{i+6}\right)=f\left(v_{i+6}\right)=1$. Denote $V_{1}=\left(P_{2} \times C_{n}\right) / \bigcup_{j=i-2}^{i+6} A_{j}$. By formula $(3.1)$, there are at most $\left[\frac{n-9+1}{2}\right]=2 k-4$ vertices in $V_{1}$, whose value are assigned -1 . With $u_{i \pm 1}$ and $v_{i+5}$, there are at most $2 k-1$ vertices in $P_{2} \times C_{n}$ whose value are assigned -1 , a contradiction.

Case 2. If $f\left(u_{i+4}\right)=-1$ or $f\left(v_{i+4}\right)=-1$, then $f\left(u_{i+5}\right)=f\left(v_{i+5}\right)=1$. Denote $V_{2}=\left(P_{2} \times C_{n}\right) / \bigcup_{j=i-2}^{i+5} A_{j}$. By formula $(3.1)$, there are at most $\left[\frac{n-8+1}{2}\right]=2 k-4$ vertices in $V_{2}$, whose value are assigned -1 . With $u_{i \pm 1}$ and $u_{i+4}\left(\right.$ or $\left.v_{i+4}\right)$, there are at most $2 k-1$ vertices in $P_{2} \times C_{n}$ that sign -1 , a contradiction.

Case 3. If $f\left(v_{i+3}\right)=-1$. Denote $V_{3}=\left(P_{2} \times C_{n}\right) / \bigcup_{j=i-2}^{i+2} A_{j}$. By formula (3.1), there are at most $\left[\frac{n-5+1}{2}\right]=2 k-2$ vertices in $V_{3}$, whose value are assigned -1 . In [4], to make $2 k-2$ vertices in $V_{3}$ whose value are assigned -1 , if and only if value -1 are assigned at the vertices $u_{i+5}, v_{i+7}, u_{i+9}, v_{i+11}, \cdots$ The remaining vertices are assigned value 1 , followed by the same rules. Because there are $n-5 \equiv 3(\bmod 4)$ vertices in $V_{3}$, it implies $f\left(u_{i-3}\right)=-1$, so $f\left[u_{i-2}\right]=f\left(u_{i-1}\right)+f\left(u_{i-2}\right)+f\left(u_{i-3}\right)+f\left(v_{i-2}\right)=0$. So 
there are at most $2 k-3$ vertices in $V_{3}$, whose value are assigned -1 . Thus, there are at most $2 k-1$ vertices in $P_{2} \times C_{n}$ t whose value are assigned -1 , a contradiction.

Based on the above claims, if $n=4 k \quad(k \geq 2)$, where $m \leq\left[\frac{n}{2}\right]-1=\frac{n}{2}-1$. So we have $\gamma_{s}^{2 n-1}\left(P_{2} \times C_{n}\right)=2 n-2 m \geq \begin{cases}n+2, & \text { if } n \equiv 0(\bmod 4) \\ 2 n-2\left[\frac{n}{2}\right], & \text { otherwise }\end{cases}$

Furthermore, the $(2 n-1)$-subdominating function $g$ of $P_{2} \times C_{n}$ is defined as follows:

(i) If $n=4 k+1$, let $A=\left\{u_{1}, u_{3}, v_{5}, \cdots, u_{4 i-1}, v_{4 i+1}, \cdots, u_{n-2}\right\}$, then $|A|=2 k=\left[\frac{n}{2}\right]$;

(ii) If $n=4 k+2$, let $A=\left\{u_{1}, u_{3}, v_{5}, \cdots, u_{4 i-1}, v_{4 i+1}, \cdots, v_{n-1}\right\}$, then $|A|=2 k+1=\left[\frac{n}{2}\right]$

(iii) If $n=4 k+3$, let $A=\left\{u_{1}, u_{3}, v_{5}, \cdots, u_{4 i-1}, v_{4 i+1}, \cdots, v_{n-2}\right\}$, then $|A|=2 k+1=\left[\frac{n}{2}\right]$

(iv) If $n=4 k$, let $A=\left\{u_{1}, u_{3}, v_{5}, \cdots, u_{4 i-1}, v_{4 i+1}, \cdots, v_{n-3}\right\}$, then $|A|=2 k-1=\frac{n}{2}-1$.

Let $g(v)=\left\{\begin{array}{ll}-1, & \text { if } \mathrm{v} \in \mathrm{A} ; \\ 1, & \text { otherwise, }\end{array}\right.$ then we have $\gamma_{s}^{2 n-1}\left(P_{2} \times C_{n}\right) \leq \begin{cases}n+2, & \text { if } n \equiv 0(\bmod 4) ; \\ 2 n-2\left[\frac{n}{2}\right], & \text { otherwise. }\end{cases}$

Consequently, the equality holds.

\section{Signed $(3 n-1)$ - subdomination number of graph $P_{3} \times P_{n}$}

Graph $P_{3} \times P_{n}$ is a grid diagram with three rows and $n$ columns.(see Figure 3) The vertex sets on the first, second, and third rows are denoted as $U=\left\{u_{1}, u_{2}, \cdots, u_{n}\right\}, V=$ $\left\{v_{1}, v_{2}, \cdots, v_{n}\right\}$ and $W=\left\{w_{1}, w_{2}, \cdots, w_{n}\right\}$. The column $i$ is denoted as $A_{i}=\left\{u_{i}, v_{i}\right.$, $\left.w_{i}\right\}(1 \leq i \leq n)$.
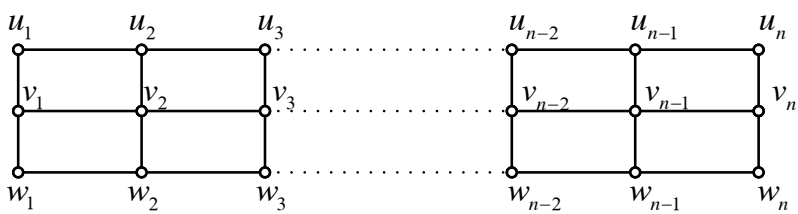

Figure 3. Graph $P_{3} \times P_{n}$. 
Suppose that $v \in P_{3} \times P_{n}$, where $f[v] \leq 0$. Because this graph is symmetric, for vertex $v$, we have the following results:

(1) If $v=u_{1}$, then $f\left(u_{1}\right)=1, f\left(u_{2}\right)=f\left(v_{1}\right)=-1$;

(2) If $v=v_{1}$, then $f\left(v_{1}\right)=1, f\left(u_{1}\right)=f\left(w_{1}\right)=-1$;

(3) If $v=u_{i}(2 \leq i \leq n-1)$, then $f\left(u_{i}\right)=1, f\left(u_{i-1}\right)=f\left(u_{i+1}\right)=-1, f\left(v_{i}\right)=1$;

Or $v=u_{i}(2 \leq i \leq n-2)$, then $f\left(u_{i}\right)=1, f\left(u_{i-1}\right)=1, f\left(v_{i}\right)=f\left(u_{i+1}\right)=-1$;

Or $v=u_{i}(3 \leq i \leq n-1)$, then $f\left(u_{i}\right)=1, f\left(u_{i-1}\right)=f\left(v_{i}\right)=-1, f\left(u_{i+1}\right)=1$;

Or $v=u_{i}(2 \leq i \leq n-1)$, then $f\left(u_{i}\right)=f\left(v_{i}\right)=-1, f\left(u_{i-1}\right)=f\left(u_{i+1}\right)=1$;

(4) $v$ cannot be $v_{2}$;

(5) If $v=u_{i}(3 \leq i \leq n-2)$, then $f\left(u_{i}\right)=1, f\left(u_{i-1}\right)=f\left(u_{i+1}\right)=f\left(v_{i}\right)=-1$;

(6) If $v=v_{3}(3 \leq i \leq n-2)$, then $f\left(v_{i-1}\right)=f\left(v_{i}\right)=f\left(v_{i+1}\right)=-1, f\left(u_{i}\right)=$ $f\left(w_{i}\right)=1$.

Consequently, we have the following lemma.

Lemma 4.1 (1) $\gamma_{s}^{3 n-1}\left(P_{3} \times P_{3}\right)=3$;

(2) $\gamma_{s}^{3 n-1}\left(P_{3} \times P_{5}\right)=5$;

(3) $\gamma_{s}^{3 n-1}\left(P_{3} \times P_{8}\right)=10$.

Theorem 4.2 For any integer $n \geq 2$ and $n \neq 3,5,8, \gamma_{s}^{3 n-1}\left(P_{3} \times P_{n}\right)=n+2\left\lceil\frac{n-2}{5}\right\rceil$.

Proof. Let vertex set $P=\left\{v \in P_{3} \times P_{n} \mid f(v)=1\right\} \quad$ and $M=\left\{v \in P_{3} \times P_{n} \mid f(v)=-1\right\}$, the order of them is denoted by $p=|P|, m=|M|$, where $p+m=3 n$. We use mathematical induction for $n$.

If $n$ is $2,4,6,7,9,10,11,12,13$, we have the $(3 n-1)$ - subdomination numbers of $P_{3} \times P_{n}$ are $2,3,5,6,7,8,9,10,10$, all of them satisfy $m \leq n-\left\lceil\frac{n-2}{5}\right\rceil$.

Suppose $n=l \geq 14$, where $m \leq l-\left\lceil\frac{l-2}{5}\right\rceil$.

If $n=l+5$. In [4], we know that $\left|\left(A_{l+1} \cup A_{l+2} \cup \cdots \cup A_{l+5}\right) \cap M\right| \leq 4$, note that $\left|\left(A_{1} \cup A_{2} \cup \cdots \cup A_{l}\right) \cap M\right| \leq l-\left\lceil\frac{l-2}{5}\right\rceil$, we have

$$
m=|M| \leq 4+l-\left\lceil\frac{l-2}{5}\right\rceil=l+5-\left\lceil\frac{l+5-2}{5}\right\rceil=n-\left\lceil\frac{n-2}{5}\right\rceil .
$$

So, $\gamma_{s}^{3 n-1}\left(P_{3} \times P_{n}\right)=3 n-2 m \geq n+2\left\lceil\frac{n-2}{5}\right\rceil$.

Furthermore. If $n \geq 2$, and $n \neq 3,5,8$. The $(3 n-1)$ - subdominating function $g$ of $P_{3} \times P_{n}$ is defined as follows:

(i) If $n=5 k+2$, let $A=\left\{u_{1}, w_{1}, v_{3}, u_{5}, w_{5}, v_{7}, \cdots, v_{5 i-2}, u_{5 i}, w_{5 i}, v_{5 i+2}, \cdots, v_{5 k-2}\right.$, 
$\left.u_{5 k}, w_{5 k}, v_{5 k+2}\right\}$, it implies that $|A|=4 k+2=n-\left\lceil\frac{n-2}{5}\right\rceil$;

(ii) If $n=5 k+3(k \neq 0,1)$, let $A=\left\{u_{1}, w_{1}, v_{3}, u_{5}, w_{5}, v_{7}, \cdots, v_{5 i-2}, u_{5 i}, w_{5 i}, v_{5 i+2}\right.$,

$\left.\cdots, v_{5 k-2}, u_{5 k}, w_{5 k}, v_{5 k+2}\right\}$, it implies that $|A|=4 k+2=n-\left\lceil\frac{n-2}{5}\right\rceil$;

(iii) If $n=5 k+4$, let $A=\left\{u_{1}, w_{1}, v_{3}, u_{5}, w_{5}, v_{7}, \cdots, v_{5 i-2}, u_{5 i}, w_{5 i}, v_{5 i+2}, \cdots, v_{5 k+3}\right\}$, it implies that $|A|=4 k+3=n-\left\lceil\frac{n-2}{5}\right\rceil$;

(iv) If $n=5 k(k \geq 2)$, let $A=\left\{u_{1}, w_{1}, v_{3}, u_{5}, w_{5}, v_{7}, \cdots, v_{5 i-2}, u_{5 i}, w_{5 i}, v_{5 i+2}, \cdots\right.$, $\left.v_{5 k-2}, u_{5 k}\right\}$, it implies that $|A|=4 k=n-\left\lceil\frac{n-2}{5}\right\rceil$;

(v) If $n=5 k+1(k \geq 1)$, let $A=\left\{u_{1}, w_{1}, v_{3}, u_{5}, w_{5}, v_{7}, \cdots, v_{5 i-2}, u_{5 i}, w_{5 i}, v_{5 i+2}, \cdots\right.$,

$\left.v_{5 k-2}, u_{5 k}, w_{5 k}\right\}$, it implies that $|A|=4 k+1=n-\left\lceil\frac{n-2}{5}\right\rceil$.

Let $g(v)=\left\{\begin{array}{ll}-1, & \text { if } \mathrm{v} \in \mathrm{A} ; \\ 1, & \text { otherwise, }\end{array}\right.$ then we have $\gamma_{s}^{3 n-1}\left(P_{3} \times P_{n}\right) \leq n+2\left\lceil\frac{n-2}{5}\right\rceil$.

Consequently, the equality holds.

\section{References}

1. J.A. Bondy, U.S.R.Murty, Graph Theory with Applications [M]. London: Macmillan, 1977.

2. E.J. Cockayne, C.M. Mynhardt, On a Generalization of Singed Dominating Functions of Graphs [J], Ars. Combin., 43 (1996).

3. Xuedong Zhang, Signed Domination Number in a Ladder $P_{2} \times P_{n}$ and a Circular Ladder $P_{2} \times C_{n}[\mathrm{~J}]$, Journal of Henan Institute of Education(Natural Science). 13(4) (2004).

4. Baogen Xu, Junxia Zhang, Guang Li, Signed Domination Numbers for Two Classes of Product Graphs [J], Journal of Henan University of Science and Technology(Natural Science), 41(2) (2020) 